\title{
Come together
}

\author{
We must do more to encourage and support interdisciplinary research approaches to energy challenges.
}

T he challenges facing our energy systems are incredibly complicated. In many cases the solutions have been - and continue to be - provided by researchers working in traditional disciplines. They may be inspired by (or work in tandem or relay-style with) colleagues from other schools, but on the whole their efforts can be siloed from those of others.

Yet a vast swathe of urgent problems are more complex - typically socio-techno-economic - issues. These multi-hyphenate challenges require more intertwined approaches that can be offered by multi- and interdisciplinary working. As we find ourselves looking deeper into questions of, for example, rapid technology development and deployment, consumer adoption, or regulatory and system revision, we will increasingly need to recognize the value of integrated research approaches.

Multidisciplinarity in energy research is fairly commonplace today. It typically sees different disciplines coming together to solve a problem, but still largely working in individual silos with their own methods and approaches, exchanging necessary information at key points in a project.

Interdisciplinarity is more complex. It typically calls for an integration of diverse traditions, methods, theories, data and concepts to solve problems in more holistic ways. It requires more silo-crossing and mutual exchanges of perspective.

The potential rewards for energy research from interdisciplinarity are great. Interdisciplinarity can enable new ways of thinking about problems that simply aren't possible otherwise, and open avenues for more creative problem solving. It can highlight problems previously hidden and reveal opportunities that may be of benefit for all.

This month's issue of Nature Energy features such an example. In their Article, Hedda Ransan-Cooper, Björn C. P. Sturmberg and colleagues at the Australian National University combine social science and engineering perspectives to examine the impacts of control algorithm design on the operation of neighbourhood-scale batteries. By characterizing the perceived risks and benefits of different stakeholders, the researchers were able to design different battery control algorithms to meet diverse needs - not just techno-economic ones. The results highlight the considerable variations in outcome - and the necessary trade-offs - that arise from thinking about all the system actors. As Christine Milchram comments in her accompanying News \& Views the work reminds us that the optimal design of a process might not be the one that the original designer was considering - a range of values need to be considered.

Happily, the value of interdisciplinary research for energy is increasingly being seen. Funders are issuing calls for greater numbers of interdisciplinary research projects, while large-scale and global initiatives are looking to establish or incorporate interdisciplinary thinking into their work. More universities are creating interdisciplinary institutes or departments, and young researchers are increasingly keen to work outside of traditional silos to tackle societal challenges.

Nonetheless, interdisciplinary research faces many difficulties. Forming collaborations in the first place remains a key challenge. Institutional structures largely remain organized along disciplinary boundaries, meaning they struggle to accommodate interdisciplinary researchers or to foster the kind of interactions required. Similarly, the organization of many networks and conferences is still siloed, reducing opportunities for engagement across domains.

Once a collaboration is formed, it faces an array of language barriers. Interdisciplinary research demands working intimately across the disciplines involved, which typically have distinct terminology. Understanding must be reached on key concepts and terms to enable teams to work collectively and effectively. This requires more direct communication than disciplinary research, often through more meetings and workshops, and can take a long time to accomplish. The language barrier reappears when trying to communicate externally about a study to broad and diverse - and likely non-interdisciplinary - audiences. Once the work has been written up and submitted to a journal, it may face difficulty in passing the judgement of editors and peer reviewers unfamiliar with the challenges of interdisciplinary work.

A further challenge for interdisciplinary research arises in terms of academic career paths. The reward and promotion structures of traditional universities and departments all too often reinforce conventional silos and struggle to recognize interdisciplinary approaches. The additional time that interdisciplinary projects by necessity take can penalize $\mathrm{PhD}$ or early-career researchers who have not yet established themselves. Evaluation panels may be unfamiliar with the different skills and knowledge required for interdisciplinary work and so they may not give it appropriate credit against standard metrics.

These and other challenges are not insurmountable and there are many examples where change is happening. A number of factors can help smooth the way for interdisciplinary work.

For instance, there needs to be more recognition that interdisciplinary research takes longer and can be more complex than typical research. This recognition should extend to funding calls and researcher evaluation processes. We cannot expect interdisciplinary $\mathrm{PhD}$ or postdoctoral positions to produce research outcomes on the same timescales as more standard approaches.

There should also be more support at institutional levels to foster interdisciplinary networks and collaborations. Traditional structures are not always appropriate for current societal challenges. There is a lot to be learned from schools and departments that have changed their approach and started to think along more interdisciplinary lines. Interdisciplinary research doesn't need to become the norm, but it should be made easier to carry out.

Interdisciplinarity would also benefit from greater open-mindedness across the spectrum of actors. Those being called to assess interdisciplinary work - be it for funding proposals, manuscript reviews, job applications or career progression - should reflect on the context it is operating in. There won't necessarily be new breakthroughs in the part of the work that intersects with their own domain, and that's fine.

There is a great deal to be gained by embracing interdisciplinary thinking. We hope there will be further growth in such studies in the future and we encourage readers to talk and engage more widely with others in the energy space. Ultimately, we are all trying to do our part to help solve hard problems. Breaking down a few more barriers between us can only help do that more effectively.

Published online: 19 August 2021 https://doi.org/10.1038/s41560-021-00897-4 